\title{
Automatic marine sensors services discovery on IP
}

\section{networks}

\author{
Daniel Mihai Toma ${ }^{1}$, Joaquin del Rio ${ }^{1}$, Tom O'Reilly ${ }^{2}$, Antoni Manuel ${ }^{1}$ \\ ${ }^{1}$ SARTI Research Group, Electronics Dept., Universitat Politècnica de Catalunya (UPC), \\ Rambla Exposició 24, Ed. C, 08800 Vilanova i la Geltrú, +(34) 938967200. www.cdsarti.org. daniel.mihai.toma@upc.edu, \\ ${ }^{2}$ Monterey Bay Aquarium Research Institute, MBARI, USA, oreilly@mbari.org
}

\begin{abstract}
Nowadays, the studies of episodic processes in the ocean is manly done through the innovative facilities called ocean observatories which provide unprecedented amounts of power and two-way bandwidth to access and control sensor networks in the oceans. The most capable ocean observatories are designed around a submarine fiber optic/power cable connecting one or more seafloor science nodes to the terrestrial power grid and communications backhaul. In a network consisting of tens, hundreds or thousands of marine sensors, manual configuration and integration becomes very challenging. Methods are required which support this task to minimize the administration efforts. This paper addresses this issue and presents an approach for the automatic discovery of marine sensors in ocean observatories. The work provides a needed reference implementation of PUCK over TCP/IP, and suggests the potential of a set of protocols and standards that could realize true end to end "Plug and Work" capability for sensor networks.
\end{abstract}

\section{INTRODUCTION}

A sensor is defined from an engineering point of view as a device that converts a physical, chemical, or biological parameter into an electrical signal [1]. While a sensor is the most basic unit, a sensor system is an aggregation of sensors, attached to a single platform [2]. Such sensor systems are the marine sensor platforms that consist of manifold sensors. These sensors need to be connected to the shore-side infrastructure so that communication with the sensors can be established and their data can be stored, displayed, or incorporated into models and simulations. A sensor network consists of a number of spatially distributed and communicating sensor resources [3], where sensor resource is the abstract representation of sensors and sensor systems.

Knowledge of each sensor's command protocol is required to operate and acquire data from the network. Making sense of these data streams to create an integrated picture of environmental conditions requires that each sensor's data and metadata be accurately processed. However, due to the large variety of sensor protocols and sensor interfaces, most applications are still integrating sensor resources through proprietary mechanisms. This manual bridging between sensor resources and applications leads to extensive adaption effort, and is a key cost factor in large-scale deployment scenarios [4].

This issue has been the driving force for the Open Geospatial Consortium (OGC) to start the Sensor Web Enablement (SWE) initiative. Within the SWE working group a suite of standards has been developed which can be used as building blocks for a Sensor Web. SWE defines the term Sensor Web as "Web accessible sensor networks and archived sensor data that can be discovered and accessed using standard protocols and application programming interfaces" [5].

However, the SWE technologies are designed from an application-oriented perspective, and cannot meet all the necessity to dynamically integrate sensors. An on-the-fly integration of sensors into the Sensor Web with a minimum of human intervention is not straight-forward with the given methods. Currently, sensors are usually connected by manually building adapters for each pair of web service and sensor type.

Bridging this interoperability gap [6] between the Sensor Web layer and the lower-level sensor layer can be addressed by several standardization efforts. Figure 1 shows the lower-level sensor layer stack and places on their positions within the layer stack four identified middleware processes susceptible to be standardized. The four identified processes susceptible to be 
standardized are sensor detection, identification, configuration and measurements.

\begin{tabular}{|c|c|c|}
\hline Sensor Web Layer & $\begin{array}{c}\text { Sensor } \\
\text { Web } \\
\text { Infrastructures }\end{array}$ & \multirow{2}{*}{$\begin{array}{c}\text { Sensor } \\
\text { Measurements } \\
\text { Sensor } \\
\text { Configuration } \\
\text { Identification } \\
\text { Detection }\end{array}$} \\
\hline Sensor Layer & $\begin{array}{c}\text { Sensor } \\
\text { Network } \\
\text { Management } \\
\text { Systems }\end{array}$ & \\
\hline Physical Layer & $\begin{array}{c}\text { Ocean } \\
\text { Observatories } \\
\text { Infrastructures }\end{array}$ & \\
\hline
\end{tabular}

Figure 1 Lower-level sensor layer stack and middleware processes susceptible to be standardized

Use of standards to operate a sensor and, retrieve and describe its data generally simplifies sensor software development, integration, operation and data processing. An example is the IEEE 1451 family of standards [7], a universal approach to connect sensors to diverse networks and systems. Another approach is the Open Geospatial Consortium (OGC) PUCK protocol that extends the sensor firmware, and provides a means to retrieve a universally unique identifier, metadata and other information from the device itself through its communication interface [9]. Thus the observing system can automatically identify the sensor and utilize the sensor and its data when it is physically installed, eliminating the need for technicians to manually set up a logical association between physical sensor and host drivers and configuration files.

However, using the oceanographic sensors in networks such as cable ocean observatories pose a great challenges and minimizing adaptation and administration efforts is a major pre-requisite for the integration of sensor data. An approach for this problem is to use sensors with low power Ethernet interface.

This research combines previous results with a framework for sensors discovery, identification and configuration based on PUCK technologies for sensors with serial communication which are extended over IP to realize Sensor Plug \& Work in
IP networks. In Figure 2 is depicted the block diagram of an IP PUCK-enabled sensor, and the component parts of the system the Zeroconf [10], PUCK and sensor proprietary protocol. The figure also shows the possible interactions with a host device (data acquisition system) connected in the same network. Thus the host can automatically detect a PUCK-enabled sensor in the marine network and the sensor, has the ability to provide metadata to the network in a standard way. The standards protocols that are used in this approach PUCK and Zeroconf are described in the following.

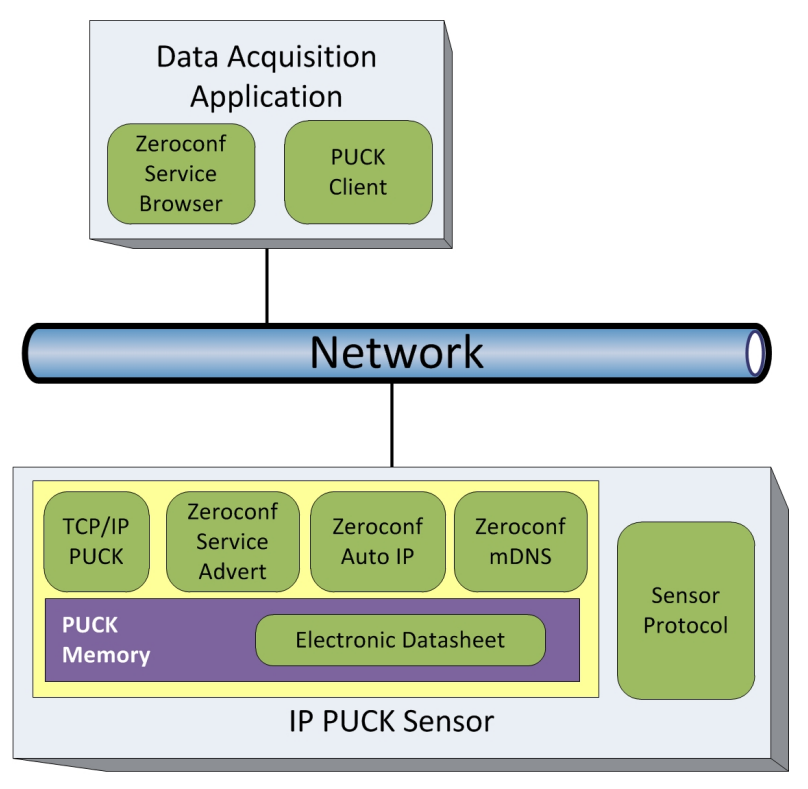

Figure 2 IP Puck Sensor Model

\section{A. PUCK PROTOCOL}

OGC PUCK defines a small "data sheet" that describes key sensor characteristics, and a standard protocol to retrieve the data sheet from the device itself. Data sheet fields include a universal serial number that is unique across all PUCKcompliant sensors. Other fields identify the sensor manufacturer and model. In addition to the data sheet, the sensor may also provide "PUCK payload" which can contain additional descriptive information (e.g. a SensorML document or IEEE 1451 TEDS), as well as actual sensor driver code. Computers on the sensor network can use PUCK protocol to retrieve this information from installed sensors and utilize it 
appropriately, e.g. to automatically identify, configure and operate the sensors, and acquire and process their data. The protocol is defined for sensors with an RS232 or Ethernet interface. Thus far PUCK has been deployed on oceanographic observatories in North America and Europe, but is generally applicable to environmental sensor networks.

\section{B. ZEROCONF PROTOCOL}

The Zeroconf component of IP PUCK provides the mechanism for automatic detection of oceanographic devices in the network. One major problem with using IP for marine sensor communication is the allocation of IP addresses, and this is also facilitated by using Zeroconf protocol. To achieve sensors ease-of-use in IP, Zeroconf provides the translation between names and IP addresses without a Domain Name Service (DNS) server. The unique sensor name is allocated by the PUCK minimal sensor datasheet and consists of sensor name and serial number.

An implementation of IP PUCK has been developed on Stellaris $\quad A R M{ }^{\circledR} \quad$ Cortex-M3 ${ }^{\mathrm{TM}}$-based microcontroller, LM3S9B96 [11]. The device it has been developed as a standard platform for oceanographic sensors, and it can work as a stand-alone sensor or as a Serial-to-Ethernet converter. In both situations a host can automatically detect the device in the network and get the sensor description using PUCK protocol.

\section{STANDARDS-BASED ARCHITECTURE FOR THE AUTOMATIC INTEGRATION OF MARINE SENSORS IN IP}

\section{NETWORKS}

The proposed Standard-based Data Management System consists of three parts that work together to automate the process. The first component is a Zeroconf browser that looks for PUCK services. When a PUCK Sensor is plugged in the network is automatically discovered by the browser that can get the IP address of the sensor, the port number of the PUCK service and the sensor name. Using the IP address and the PUCK service port number, the second component of the system, the PUCK application, it is connected with the marine sensor to retrieve the PUCK standard datasheet and/or payload. The payload can be an electronic datasheet giving detailed information about the marine sensor, the metadata of the sensor, and the communication protocol of the sensor. This document may be used by the third component of the system, the data acquisition system to send commands to the sensor to retrieve data as shown in Figure 3.

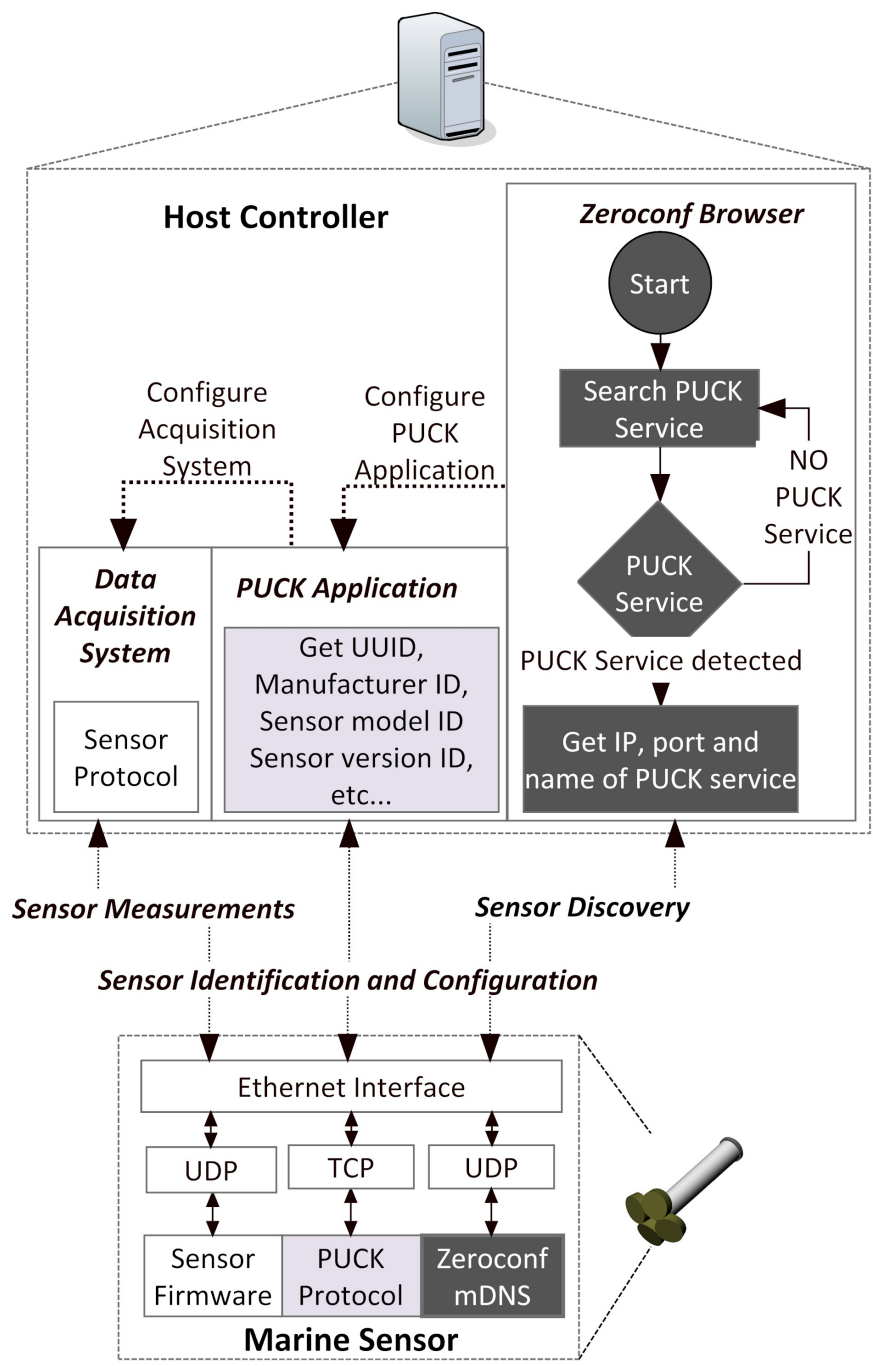

Figure 3 Integration of marine sensors in networks

\section{CONCLUSIONS}

The use of PUCK protocol with in a sensor facilitate the integration of the sensor within an observatory allowing storage of a self-descript sensor metadata in different payloads types such as IEEE1451 TEDS or SensorML. An automatic 
sensor recognition protocol has been proposed in order to enable the host to automatically detect and configure a new sensor using Zeroconf and PUCK Protocol. Our prototype sensor network demonstrates how sensors may be operated without the need to manually configure the sensor parameters such as IP address and DNS and easily can access its data both for processing or archiving or shared viewing via web.

\section{ACKNOWLEDGMENT}

This work was supported by the Spanish Ministry of Economy and Competitiveness under the research project: "Sistemas Inalambricos para la Extension de Observatorios Submarinos" (CTM2010-15459).

\section{REFERENCES}

[1] Bermudez, L.; Delory, E.; O'Reilly, T.; del Rio Fernandez, J. Ocean Observing Systems Demystified. In Proceedings of OCEANS 2009, Marine Technology for Our Future: Global and Local Challenges, Biloxi, MS, USA, October 2009; IEEE: New York, NY, USA, 2009; pp. 1-7.

[2] Stasch, C.; Janowicz, K.; Bröring, A.; Reis, I.; Kuhn, W. A StimulusCentric Algebraic Approach to Sensors and Observations. In Proceedings of 3rd International Conference on Geosensor Networks, GSN 2009, Oxford, UK, July 2009; Trigoni, N., Markham, A., Nawaz, S., Eds.; Lecture Notes in Computer Science; Springer: Berlin, Germany, 2009; Volume 5659, pp. 169-179.

[3] van Zyl, T.; Simonis, I.; McFerren, G. The Sensor Web: Systems of Sensor Systems. Int. J. Digital Earth 2009, 2, 16-30.

[4] Aberer, K.; Hauswirth, M.; Salehi, A. A Middleware for Fast and Flexible Sensor Network Deployment. In Proceedings of 32nd International Conference on Very Large Data Bases, Seoul, Korea, September 2006; Dayal, U., Whang, K.Y., Lomet, D.B., Alonso, G., Lohman, G.M., Kersten, M.L., Cha, S.K., Kim, Y.K., Eds.; ACM: New York, NY, USA, 2006; pp. 1199-1202
[5] Botts, M.; Percivall, G.; Reed, C.; Davidson, J. OGC Sensor Web Enablement: Overview and High Level Architecture. In Proceedings of GeoSensor Networks, 2nd International Conference, GSN 2006, Boston, MA, USA, October 2006; Nittel, S., Labrinidis, A., Stefanidis, A., Eds.; Lecture Notes In Computer Science; Springer: Berlin, Germany, 2008; Volume 4540, pp. 175-190.

[6] Walter, K.; Nash, E. Coupling Wireless Sensor Networks and the Sensor Observation Service-Bridging the Interoperability Gap. In Proceedings of 12th AGILE International Conference on Geographic Information Science 2009, Hannover, Germany, June 2009; Available online: http://plone.itc.nl/agile_old/Conference/2009-hannover/shortpaper.htm (accessed on 25 February 2011).

[7] Lee, K. IEEE 1451: A Standard in Support of Smart Transducer Networking. In Proceedings of 17th Sensoration and Measurement Technology Conference, Baltimore, MD, USA, May 2000; Volume 2, pp. $525-528$.

[8] Marc Nogueras, Carola Artero, Joquín del Rio, Antoni Mànuel, David Sarrià, "Control and acquisition system design for an Expandable Seafloor Observatory", IEEE OCEANS09, 11-14 May, Bremen, Germany.

[9] T.O'Reilly, K.Headley et al, "MBARI technology for self-configuring interoperable ocean observatories", Proceedings of the Marine Technology Society / Institute of Electrical and Electronics Engineers Oceans Conference, Boston, Massachusetts.

[10] Williams, A. (2002). Zeroconf. Retrieved from Zero Configuration Networking: http://files.zeroconf.org/draft-ietf-zeroconf-reqts-12.txt.

[11] Toma, D.M.; O'Reilly, T.; del Rio, J.; Headley, K.; Manuel, A.; Broring, A.; Edgington, D.; , "Smart sensors for interoperable Smart Ocean Environment," OCEANS, 2011 IEEE - Spain, vol., no., pp.1-4, 6-9 June 2011. 